livraisons

d'Histoire

de l'Architecture

\section{Livraisons de l'histoire de l'architecture}

14 | 2007

Piscines

\title{
La piscine municipale de Bruay-en-Artois et le socialisme municipal d'Henri Cadot
}

The swimming pool of Bruay-en-Artois and its links with local socialism

Der Bau der Badeanstalt in Bruay-en-Artois unter der sozialistischen

Stadtverwaltung Henri Cadots

\section{Marc Verdure}

\section{OpenEdition}

Journals

Édition électronique

URL : http://journals.openedition.org/lha/432

DOI : $10.4000 /$ /ha. 432

ISSN : 1960-5994

Éditeur

Association Livraisons d'histoire de l'architecture - LHA

Édition imprimée

Date de publication : 10 décembre 2007

Pagination : 95-109

ISSN : 1627-4970

\section{Référence électronique}

Marc Verdure, «La piscine municipale de Bruay-en-Artois et le socialisme municipal d'Henri Cadot»,

Livraisons de l'histoire de l'architecture [En ligne], 14 | 2007, mis en ligne le 10 décembre 2009, consulté le 30 avril 2019. URL : http://journals.openedition.org/lha/432 ; DOI : 10.4000//ha.432

Ce document a été généré automatiquement le 30 avril 2019.

Tous droits réservés à l'Association LHA 


\section{La piscine municipale de Bruay-en- Artois et le socialisme municipal d'Henri Cadot}

The swimming pool of Bruay-en-Artois and its links with local socialism

Der Bau der Badeanstalt in Bruay-en-Artois unter der sozialistischen

Stadtverwaltung Henri Cadots

Marc Verdure

«Construire un hôpital, c'est de l'assistance ;

construire un stade, c'est de la prévoyance "

Édouard Herriot ${ }^{1}$

1 L'entre-deux-guerres fut, dans le Nord-Pas-de-Calais, une période de concurrence entre les édiles politiques de gauche et les compagnies des mines, dont le paternalisme se heurtait aux préoccupations socialistes. Les nécessités de la reconstruction dans les zones ravagées par les combats, du retard à rattraper dans le logement social et de l'évolution démographique expliquent l'essor de la commande publique qui s'intéresse de plus en plus aux équipements sportifs.

2 D'autre part, cette période fut celle d'un intérêt grandissant pour le sport. Si l'on accorde au Front populaire et à Léo Lagrange le mérite du développement de ces pratiques, un examen plus précis des exemples municipaux montre que les habitudes locales précédèrent la théorisation nationale. En quoi la piscine de Bruay-en-Artois, piscine de plein air construite en 1935-1936 au cœur d'un habitat ouvrier, illustre-t-elle ce double contexte de conquête municipale et socialiste et de naissance d'un nouvel idéal sportif? 


\title{
Le contexte sociopolitique de l'entre-deux-guerres.
}

\author{
Henri Cadot et la compagnie des mines de Bruay : l'enjeu sportif
}

3 La compagnie des mines de Bruay occupa la concession de Bruay-en-Artois de 1852 à 1945 et exploitait, en 1938, quatorze puits répartis entre les communes de Bruay, Haillicourt, Divion et Houdain. Son patrimoine était immense et elle avait encerclé le cœur de Bruay d'une ceinture de cités pavillonnaires. Employant environ 18000 ouvriers à Bruay², la Compagnie était omniprésente dans la vie quotidienne des habitants. Dirigée notamment par la famille Marmottan, son influence dans la région fut très importante, elle contrôla jusqu'en 1914 la vie politique du bassin, parfois même en prenant les rênes de la vie municipale locale. Jules Marmottan fut le maire de Bruay entre 1870 et 1879.

Face à une telle omniprésence, les syndicats s'activaient, entre autres grâce à Henri Cadot dès $1889^{3}$. Comme tous les grands chefs de mineurs du Pas-de-Calais de la première génération, il mena une carrière politique parallèle à son engagement syndical : élu viceprésident du syndicat des mineurs du Pas-de-Calais en 1892, il se présenta à tous les scrutins municipaux et nationaux mais ne fut élu député qu'en 1914, puis maire en 1919. À l'Assemblée nationale et au Sénat, Cadot intervint dans tous les débats budgétaires et charbonniers, militant pour l'amélioration du sort des ouvriers mineurs. Outre cette activité parlementaire, il fut un chef syndicaliste virulent lors des diverses grèves qui secouèrent le Pas-de-Calais.

Dans ce contexte de concurrence entre la mairie socialiste et la compagnie des mines pour la maîtrise de la vie locale, il semble que les échanges furent assez vifs dans un premier temps, les socialistes n'hésitant pas à critiquer l'action délétère du patronat. La presse d'opposition ${ }^{4}$ se fit ainsi l'écho de conflits et de mots très durs prononcés par Henri Cadot à l'encontre de la Compagnie. Le sport et les loisirs firent, semble-t-il, partie des éléments de concurrence entre les deux rivaux; une des marques essentielles du pays minier pendant l'entre-deux-guerres fut en effet l'existence et la vitalité d'un grand nombre de sociétés presque toutes placées sous la présidence d'honneur des autorités minières et municipales dont elles recevaient le soutien pécuniaire. Les Marmottan versèrent ainsi un don de 50000 francs en faveur des associations musicales et sportives; la plupart des associations musicales étaient présidées par des ingénieurs des mines (à l'exception de celles créées par la municipalité), de même que quelques-unes des associations sportives. D'autre part, on remarque, à travers l'itinérance du club de football de l'Union sportive bruaysienne (USB) qui joua jusqu'en 1933 sur un stade construit par la compagnie des mines puis après cette date dans le stade municipal, l'esquisse d'une rivalité pour attirer à soi la population et un loisir populaire.

\section{L'évergétisme d'une municipalité socialiste}

Au sortir de la première guerre mondiale, la ville de Bruay-en-Artois se retrouva avec un grand nombre d'édifices dévastés, vétustes ou trop étriqués pour ses besoins. En outre, la ville dut faire face à un accroissement démographique rapide, passant de 2500 habitants en 1875 à 32000 en 1936. La plupart des édifices publics étaient inutilisables ou inexistants, sans compter le problème de l'habitat. La mairie socialiste élue en 1914, qui se maintint aux affaires pendant toute l'entre-deux-guerres, lança ainsi dès les années 1920 
un grand programme de travaux guidés par l'architecte communal de l'époque, Paul Hanote, probablement en lien avec la loi Cornudet de $1919^{5}$. Paul Hanote, aidé de son fils René, reconstruisit entièrement l'hôtel de ville en 1928-1930 dans un style néoRenaissance; une salle des fêtes fut édifiée dans un style Art déco par les mêmes architectes en 1930; des habitations à bon marché furent construites à partir de 1930. D'autres programmes plus spécifiques concernèrent les écoles et groupes scolaires, nombreux à s'édifier avec l'aide de la compagnie des mines.

7 La construction de la piscine en 1935-1936 s'inscrit ainsi dans l'ère des grandes entreprises sociales ${ }^{6}$, période où les urbanistes et édiles prirent la ville en compte dans sa globalité, en concevant de véritables "équipements ", harmonieusement insérés dans la vie urbaine. En outre, si les thèmes de l'hygiène, de la santé, du logement, des arts et de la culture furent mentionnés très tôt dans la politique locale française, celui du sport n'apparut qu'à la veille de la première guerre mondiale. Le patrimoine nautique français faisait ainsi largement défaut en $1922^{7}$ avant de progressivement s'accroître ${ }^{8}$. Jean-Paul Callède a résumé, autour de l'exemple de Bègles, le contexte de l'époque ${ }^{9}$ : le modèle d'Édouard Herriot et de sa politique en matière d'équipement sportif à Lyon ${ }^{10}$, l'influence des écrits de Tony Garnier ainsi que la redéfinition, au début des années 1930, du programme d'action sportive du parti socialiste $\mathrm{SFIO}^{11}$. Cela fut probablement un stimulant pour l'action municipale bruaysienne.

8 À travers la lecture du Bulletin municipal de Bruay-en-Artois ${ }^{12}$, document électoraliste publié par la municipalité sortante de Bruay en avril 1935, à la veille du scrutin municipal de mai, on s'aperçoit que le souci des élus socialistes, ultra-majoritaires à l'hôtel de ville, fut bien la santé, le bonheur, la vitalité de la population, « la défense de la race contre la maladie et la déformation ». Les déclarations du maire Henri Cadot en conseil municipal ${ }^{13}$ se firent l'écho des demandes des associations et la mention, dans le Bulletin, des sections de football, athlétisme, basket-ball, tennis et gymnastique, des sections sportives de l'École primaire supérieure et du Sport ouvrier bruaysien, montre l'utilité des chantiers municipaux. Le projet de piscine, aussi désigné dans le Bulletin comme une «école de natation avec piscine de plein air» (p. 4) était décrit comme la réponse de la mairie au vœu de la population, la municipalité recherchant «l'intérêt de l'hygiène et de la santé publique ».

9 Il semble que le «socialisme municipal des années $1930^{14}$ » à Bruay-en-Artois ait fait son miel de ce contexte interventionniste. Ce socialisme d'action, dominé par le souci de servir les intérêts généraux de la cité par delà les problèmes de rivalité idéologique nationaux, souhaitait également rompre avec une certaine inactivité et compenser le retard accumulé par une série de grands travaux à l'échelle de la commune. Ainsi, prévenant la critique possible d'immobilisme et d'opportunisme politique, qui n'eût pu manquer de surgir en considérant que la plupart des équipements urbains furent construits dans les années 1930 alors que la municipalité socialiste était en place depuis 1914, le Bulletin évoquait la difficulté d'aménager une cité ancienne, à l'espace contraint et son souhait d'intervenir « sans tapage avec le souci du Bien public » (p. 4).

\section{Les critiques de l'action municipale}

10 «Vieux retraité du parlementarisme $»^{15}$, Henri Cadot fut volontiers critiqué par la presse locale. Le Journal de Bruay lança ainsi en mai 1935 une longue campagne de dénigrement et d'accusations à l'égard des socialistes et de leur chef local, le principal reproche étant son 
ambiguïté à l'égard de la compagnie des mines de Bruay, qu'en tant qu'ancien mineur, Cadot surveillait avec attention. Citant le Bulletin présenté la veille des élections municipales de 1935, le Journal s'interrogeait sur le financement des grands chantiers, passé sous silence, à l'exception notable de celui de la piscine, payée par emprunt ${ }^{16}$. Le journal estimait à trente millions de francs le montant des travaux, presque intégralement financés par la Compagnie. En fin de compte, il soulignait l'hypocrisie des socialistes qui disqualifiaient auprès de l'opinion publique ceux auprès desquels ils obtenaient ensuite le financement de leurs projets. L' " homme des trente deniers » admit par la suite un emprunt de cinq millions de francs auprès de la Compagnie mais il est probable que les opposants eurent une responsabilité dans sa non-réélection au fauteuil de sénateur en 1935. Il faut toutefois noter que les édifices sportifs, payés par emprunt auprès de la caisse des dépôts et par une subvention de l'État, ne furent jamais critiqués, ni au moment de leur présentation au public dans la presse, ni après leur ouverture. Il faut probablement en conclure que la contestation de ces équipements ne recevait pas un grand écho auprès de la population.

\section{La piscine communale de Bruay-en-Artois et le nouvel idéal sportif}

\section{Le Nord de la France et le développement des sciences sportives}

11 Dès le début du XXe siècle, le Nord de la France se montra pionnier, en organisant des compétitions et des clubs de natation dans les canaux (Tourcoing). Si les premiers bassins aux normes olympiques $(50 \mathrm{~m})$ furent édifiés à Paris et en région parisienne, le Nord ne recevant sa piscine olympique qu'en 1933 (à Tourcoing encore), la pédagogie se développa grâce à l'activisme d'un maître-nageur ingénieur, Paul Beulque. Jusqu'à cette époque, la leçon collective était souvent difficile dans un espace qui ne comportait pas de petite profondeur, et peu nombreuses étaient les piscines conçues dès l'origine avec un espace spécifique pour l'apprentissage ${ }^{17}$. Si l'idée du petit bain (ou " petite profondeur ») existait dès la fin du XIXe siècle, il était rarement destiné aux débutants. La règle était plutôt à la petite piscine à profondeur progressive, on n'imaginait pas encore alors de différencier systématiquement le bassin sportif du bassin d'apprentissage. Une véritable pratique scolaire vit néanmoins le jour à Tourcoing grâce à l'action combinée du sénateur maire Gustave Dron et de Paul Beulque, dès 1911. Ce dernier inventa ainsi une potence montée sur une poutre transversale au bassin susceptible de permettre à l'élève de répéter les mouvements appris à sec sur le bord du bassin. Un tel type de potence existait, au témoignage des usagers, à Bruay-en-Artois.

Concernant le cas de Bruay-en-Artois, il est intéressant de noter que la piscine portait aussi le nom d' "école de natation ", soulignant ainsi le souci pédagogique des maitres d'ouvrage ; un bassin était même entièrement destiné au public scolaire et débutant. Néanmoins, la mention répétée des "attentes" de la population pose question, car il n'existait pas de club avant la création de la piscine ${ }^{18}$ : le club nautique bruaysien fut créé en 1936, l'année de l'inauguration, et le club nautique franco-polonais « Les Requins » vit le jour en 1937 ; ce dernier réclama rapidement l'accès privilégié à la piscine pour ses entraînements. Il semble ainsi que l'offre ait occasionné la demande... 


\section{La piscine, une œuvre hygiéniste et sociale} sportive, il faut élargir le propos à l'ensemble du stade-parc ${ }^{19}$, dans lequel s'intégra le projet de la piscine (ill. 1), et lire les déclarations de l'architecte justifiant les buts poursuivis ${ }^{20}$ :

Encourager et favoriser le développement du sport en général. Permettre à la jeunesse d'occuper sainement ses loisirs. Permettre à tous de respirer un air assaini. Encourager les parents à faire donner l'éducation physique à leurs enfants. Prêter un concours efficace aux sociétés sportives en mettant à leur disposition des terrains ${ }^{21}$.

III. 1 : Plan d'ensemble du stade-parc comportant la piscine municipale

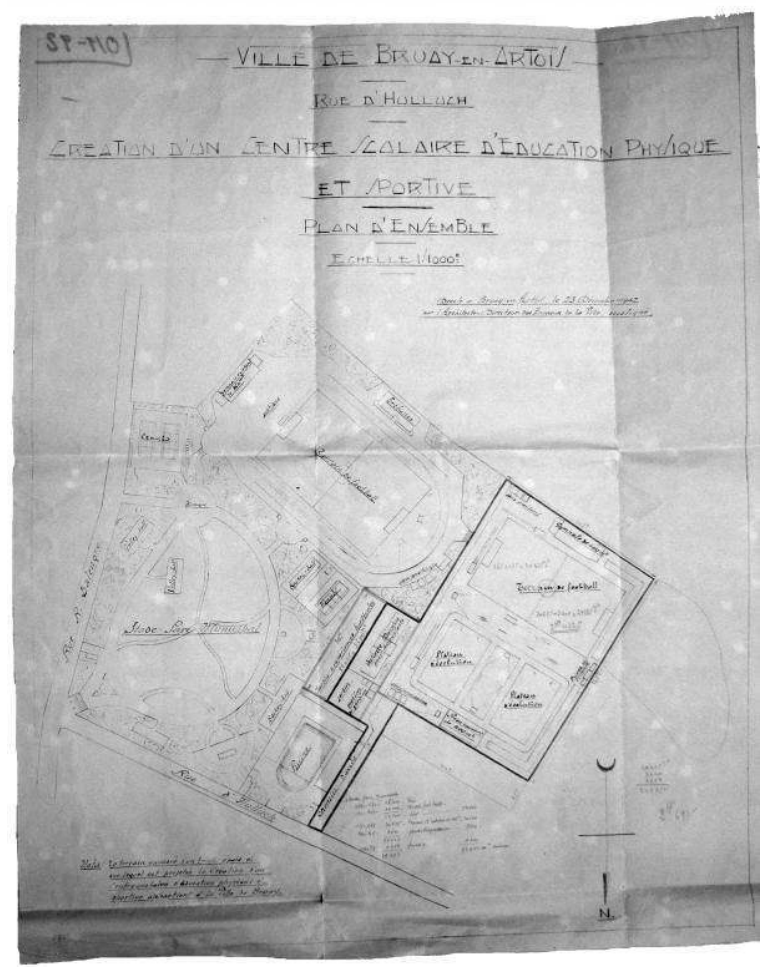

1942

CL. DE L'AUTEUR

On a déjà souligné précédemment combien l'enjeu sportif pouvait occasionner de rivalités entre la commune et la compagnie des mines : accueillir le club de football fondé en 1906 (union sportive bruaysienne), la section d'athlétisme du sport ouvrier bruaysien, le lawntennis club créé en 1925, les sociétés de gymnastiques, organiser le championnat d'athlétisme du Nord Pas-de-Calais en juillet 1935, permit à la commune de mettre en pratique, bien avant l'heure, l'adage de Léo Lagrange : « Notre but, simple et humain, est de permettre aux masses de la jeunesse de trouver dans la pratique des sports la joie et la santé2 $^{22}$.» La création de la piscine, qui vient combler une " lacune inconcevable », permit ainsi à la municipalité sortante d'éviter à la population « de chercher ailleurs que dans la Cité la satisfaction de [son] sport favori ». 

Pas-de-Calais prit alors l'initiative en février 1932 de financer à Bruay des chantiers employant une cinquantaine de chômeurs, ceux-ci étant payés par le département dans une proportion de $58 \%$. Les travaux concernèrent ainsi l'aménagement et le terrassement, la construction de murs de soutènement et de clôtures, les entrées du terrain de football et des vestiaires. Il en fut de même pour la piscine : le cahier des charges particulières, clauses et conditions générales du 27 janvier 1935, stipulait (article 11) que «l'employeur [serait] tenu d'employer exclusivement sur son chantier la main d'œuvre non spécialisée de la commune de Bruay-en-Artois ${ }^{23}$.

Le coût du chantier de construction de la piscine, estimé à 971560 francs lors du conseil municipal du 15 février 1935, fut couvert, comme le précisait le Bulletin de 1935, par un emprunt de 650000 francs auprès de la caisse des dépôts et consignations et par une subvention de 200000 francs de l'État. Le droit à cette dernière se justifia en raison de la vocation sociale de l'entreprise: dans sa correspondance avec l'État, la ville de Bruay rappela sans cesse qu'il s'agissait d'accéder à la demande d'une population de 32000 habitants et de lutter contre le chômage en favorisant la renaissance économique. La multiplication des édifices sportifs, guidée tout d'abord par des intérêts privés puis par ceux des pouvoirs publics, ouvrait en effet un marché nouveau à une économie de la construction en perte de vitesse ${ }^{24}$.

\section{L'ouverture de la piscine : les usages}

Après sept mois de travaux, le $1^{\text {er }}$ août 1936, eut lieu l'inauguration de la piscine en même temps que le baptême officiel de plusieurs nageurs, en présence du député-maire Henri Cadot, de son collègue député Gaston Beltrémieux et de l'architecte Paul Hanote ${ }^{25}$.

La piscine était prévue pour accueillir environ un millier de nageurs par jour, les prix d'entrée devant être les plus bas possibles. Le centre de l'école de natation est occupé par la piscine composée de deux bassins séparés par une claire-voie (ill. 2). 
III. 2 : Projet de construction d'une école de natation avec bassin en plein air

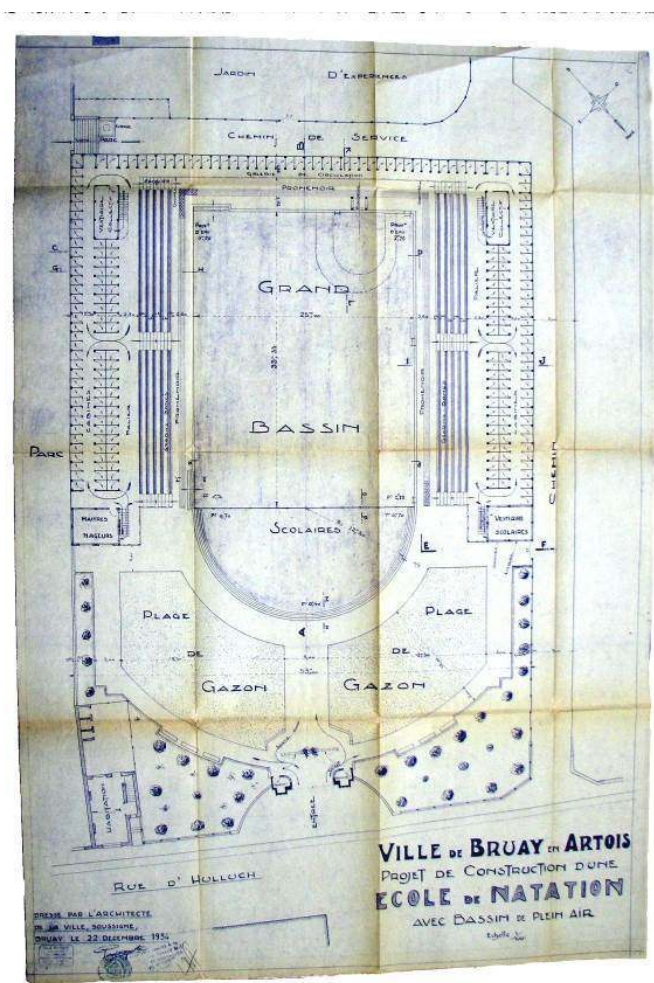

Plan de 1934

CL. DE L'AUTEUR

L'un est rectangulaire de $25 \times 33,33 \mathrm{~m}$ et l'autre pour scolaires en demi-cercle de 12,5 m de rayon. Le grand bassin rectangulaire, en pente dans le sens de la largeur (de 0,7 $\mathrm{m}$ à 2,2 $\mathrm{m}$ ), accueille une fosse à plongeon dotée de quatre plongeoirs superposés (ill. 3).

\section{3 : École de natation, vue d'ensemble des bassins}

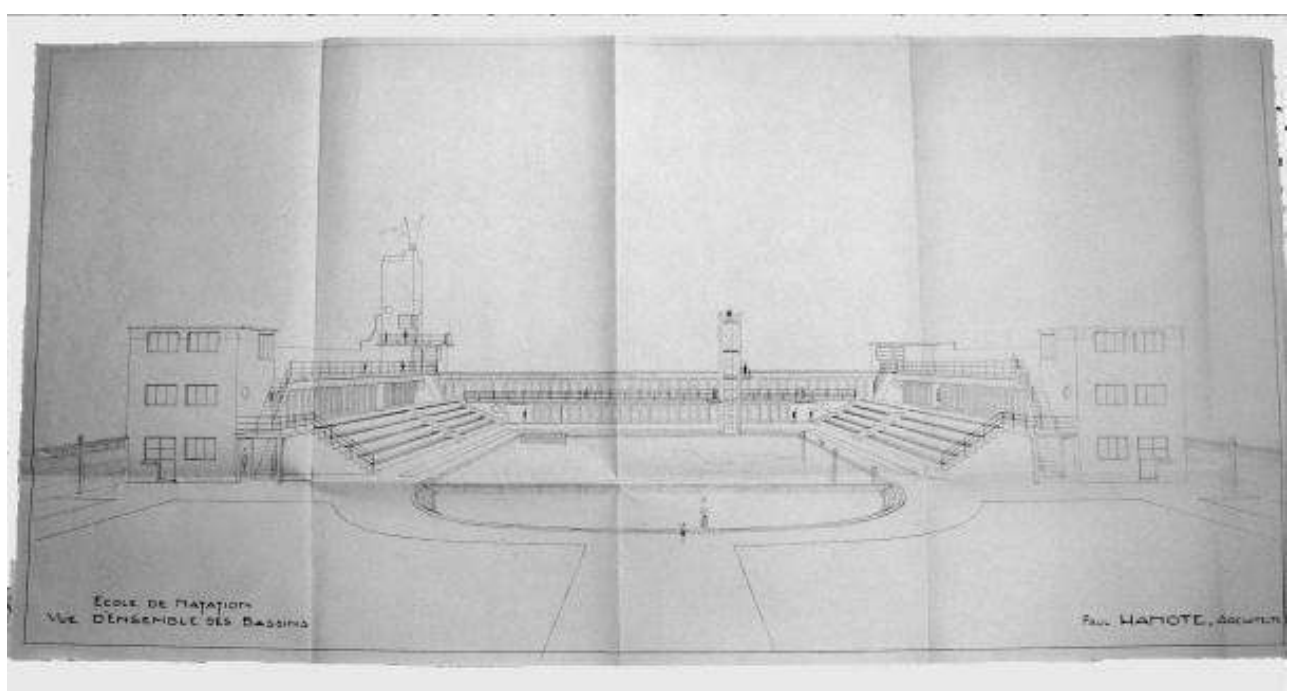

1935

CL. DE L'AUTEUR 
21 Les deux bassins sont carrelés de panneaux de céramique bleue, afin de faciliter le nettoyage et de donner à l'eau un aspect agréable.

22 Les 246 cabines (ill. 4) et les quatre vestiaires collectifs sont établis sur trois épaisseurs sur les longs côtés du bassin et sur un unique rang dans le fond (cabines pour deux personnes).

\section{4 : Couloir d'accès aux cabines}

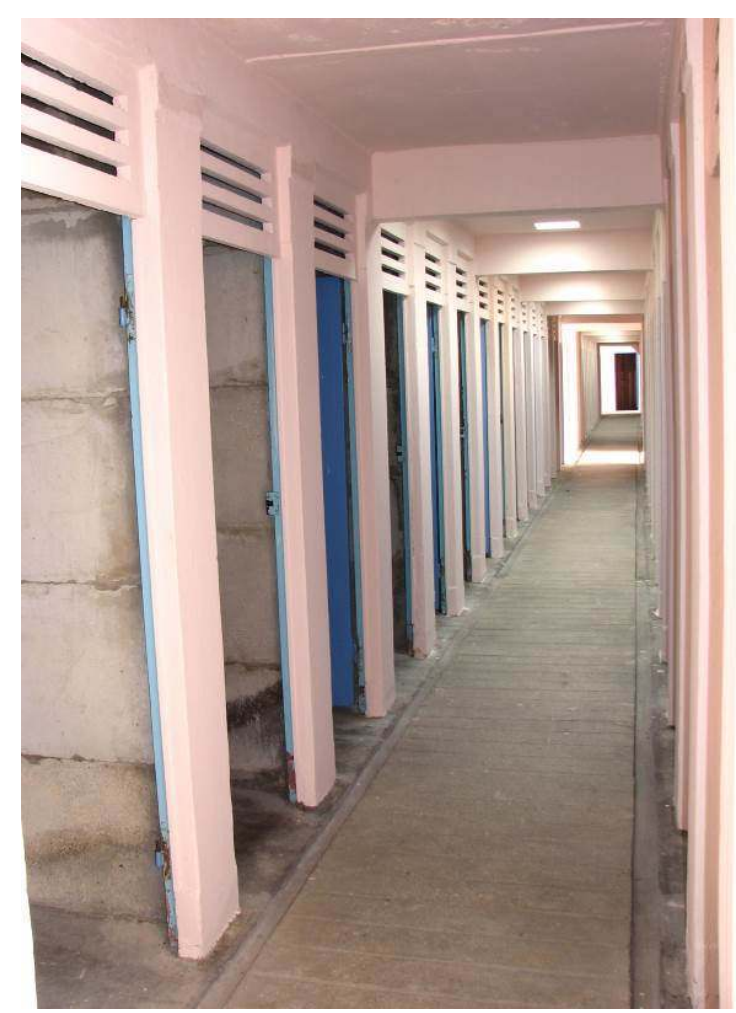

2007

CL. DE L'AUTEUR.

23 Les deux ailes sont surmontées d'une terrasse "solarium ». À l'angle de deux ailes est installé un poste de secours surmonté d'un réservoir (ill. 5). 


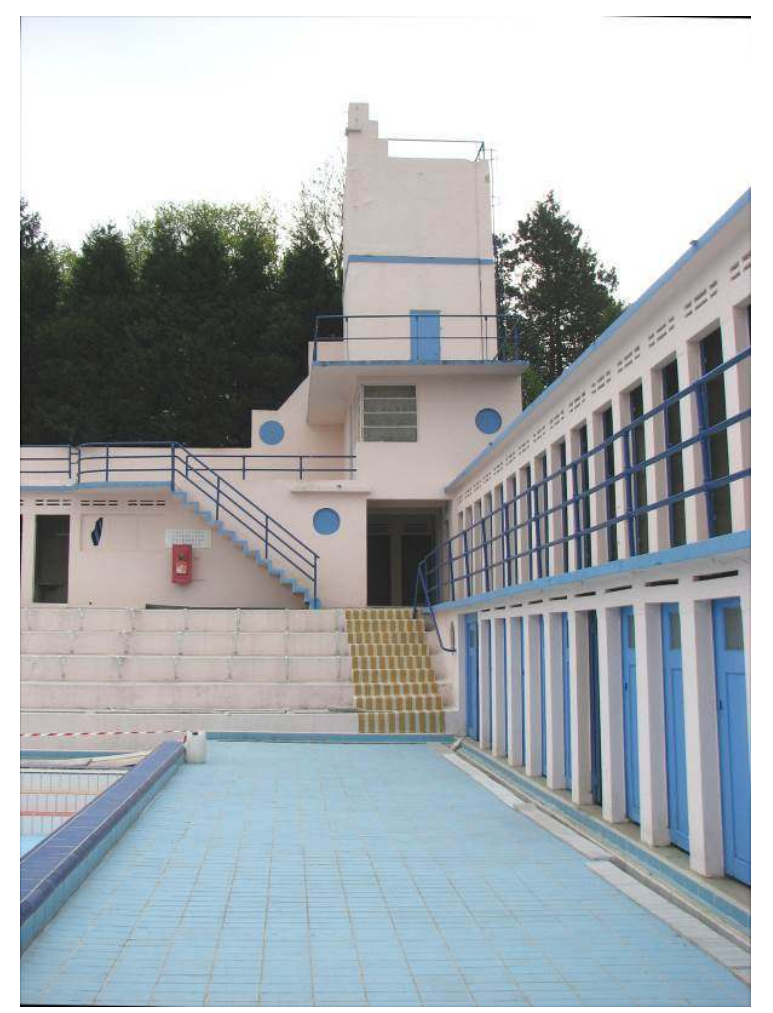

2007

CL. DE L'AUTEUR

Les bassins, entourés d'un promenoir dallé et de gradins, ne sont accessibles qu'aux baigneurs préalablement passés par la « douche de propreté ». L'alimentation en eau des bassins provient d'un forage accordé par la compagnie des mines. Le parti est donc traditionnel, notamment dans la disposition des cabines de déshabillage autour du bassin de nage et, dans le passage, par un pédiluve avant d'entrer dans le bassin.

\section{Une réalisation architecturale fonctionnelle}

La construction de la piscine s'inscrit dans un projet global qui inclut plusieurs équipements sportifs ainsi qu'un espace vert de détente. Les préoccupations sont donc bien davantage celles d'un urbaniste que d'un architecte et il ne faut pas voir dans le chantier de la piscine de Bruay-en-Artois un manifeste architectural comme celui de la piscine de Bègles. À Bruay, comme à Bordeaux ou à Lyon, l'édifice est fonctionnel et rationnel, utilisant les matériaux modernes et un vocabulaire simple et dépouillé. L'architecte a ainsi prévu, comme beaucoup de ses confrères à la même époque (Léon Madeline à Bordeaux), l'installation de locaux techniques (buanderie, réservoirs d'eau, filtrage de l'eau) et l'emploi de matériaux simples, d'entretien facile, peu onéreux et résistant à toute épreuve. Le cahier des charges particulières, clauses et conditions générales du 27 janvier 1935 soulignait ainsi que les matériaux de construction (sable, ciment, briques, bois, fers, peinture, vitrerie) devaient être de "première qualité et de premier choix », agréés par le maître d'œuvre ${ }^{26}$. 
Le parti général évoque immédiatement celui d'un navire, dans l'élévation basse et rythmée par les supports verticaux des ailes en retour, la couleur pâle de la peinture, la présence d'une tour semblable à la cheminée d'un transatlantique et le choix d'oculi décoratifs faisant penser à des hublots. Quelques détails évoquent en outre le mouvement moderne international, qui se développe dans les années 1920-1930 à partir des recettes de l'Art déco : le portique d'accès sur la rue (ill. 6), le dessin des kiosques d'entrée (ill. 7), l'aspect général des façades sur les bassins, qui mêlent à peu de frais ampleur et monumentalité (ill. 8).

\section{6: Portique d'accès sur la rue}

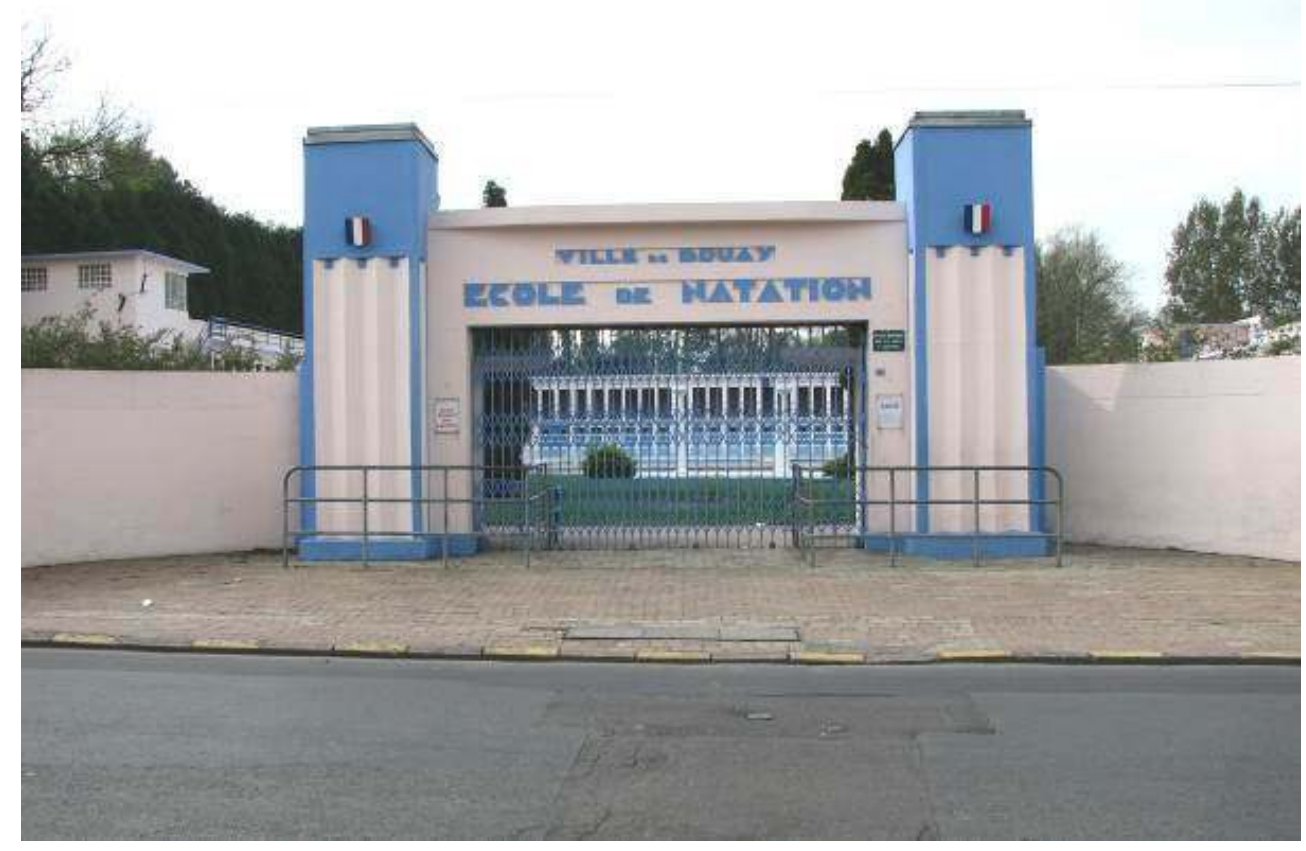

2007

CL. DE L'AUTEUR 
III. 7 : Kiosque d'entrée

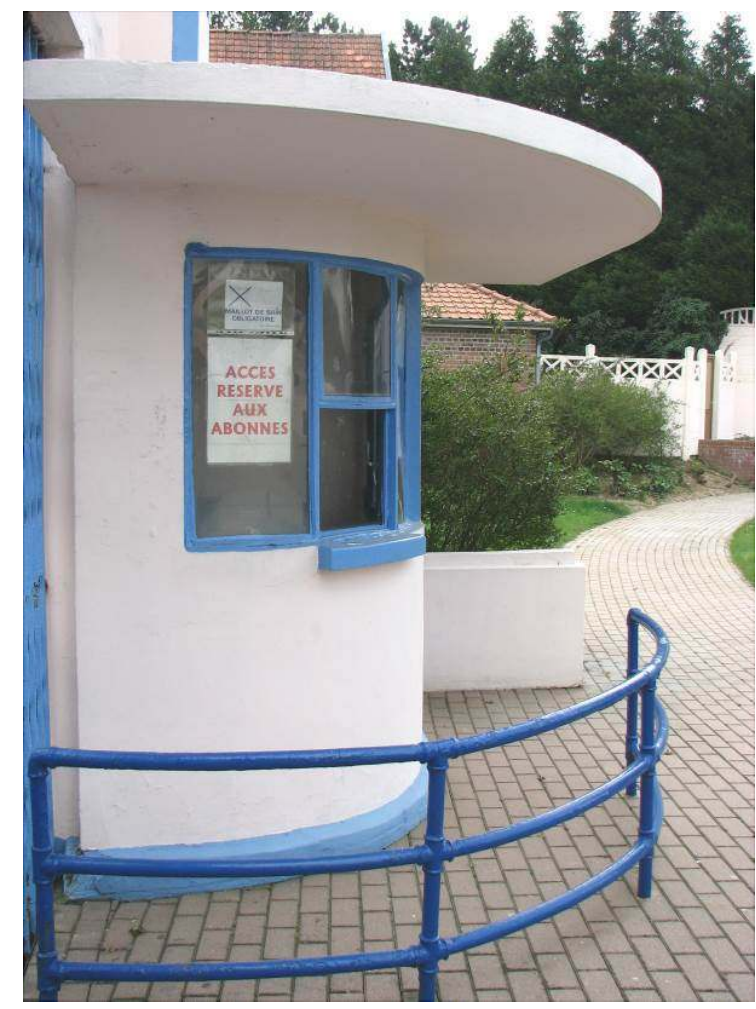

2007

CL. DE L'AUTEUR 


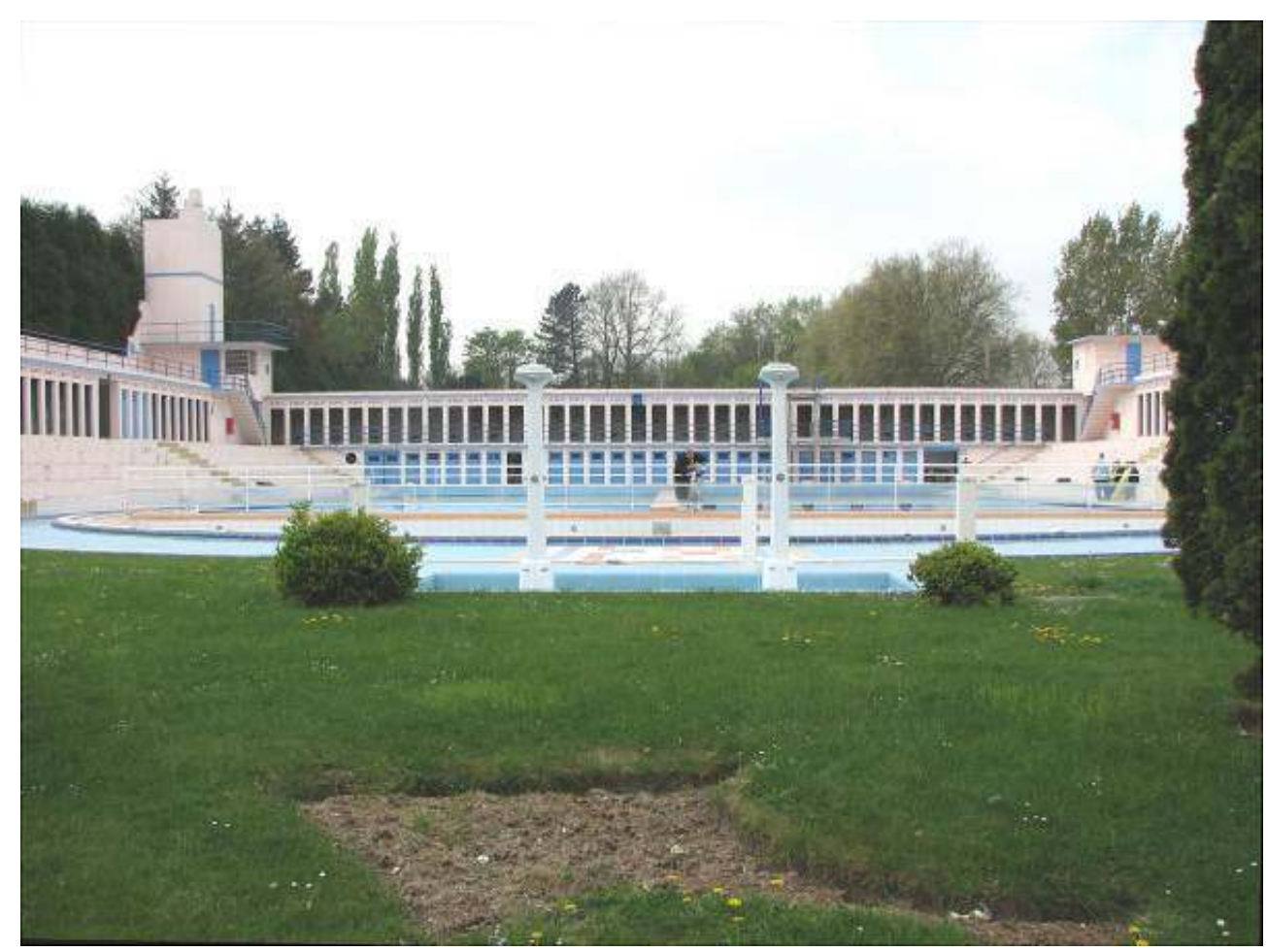

2007

CL. DE L'AUTEUR

Paul Hanote ne cherchait probablement pas à s'inscrire dans un style ; l'eût-il voulu, cela aurait été compromis par l'utilisation d'une main d'œuvre peu qualifiée. Comme beaucoup d'architectes urbanistes de cette époque, qui trouvaient à s'exprimer dans les revues spécialisées ${ }^{27}$, il mêla le goût de l'équipement public à celui de l'œuvre sociale. Parmi ses sources possibles figure la piscine construite à Madrid en 1932 par Luis

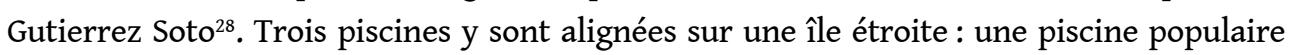
en plein air et deux piscines de luxe couvertes et chauffées, accompagnées sur toute leur longueur de cabines. Les deux piscines de luxe, mitoyennes, ont la même forme que les bassins de Bruay (rectangle et demi-cercle). Enfin, les couleurs utilisées, le motif du hublot, le parti général ${ }^{29}$ rappellent la métaphore navale. On peut plus généralement rapprocher l'étirement monotone des bâtiments de cabines de Bruay du modèle de la plage, souvent d'élévation basse (plage du Lys-Chantilly, 1934; Meaux-Trielport, Wiesbaden) ; l'idée des « solariums » sur les terrasses emprunte d'ailleurs aussi à ce type de construction. Mais le plongeoir, qui représente souvent un morceau de bravoure (Bordeaux, Trouville), n'est pas particulièrement mis en valeur à Bruay.

\section{Des partis pris esthétiques spécifiques?}

Situé au cœur de plusieurs cités minières, l'ensemble se voulait probablement un manifeste de la présence municipale, une preuve de son souci du bien-être des habitants. Le parti pris esthétique des bâtiments n'est donc pas innocent. Paul Hanote ${ }^{30}$, qui travailla à Bruay et aux environs pendant presque toute l'entre-deux-guerres, semble avoir développé deux styles en fonction de la commande, l'un traditionnel et l'autre plus 
moderne. Pour les hôtels de ville de Bruay en 1930 (avec son fils René ${ }^{31}$ ) et de Labuissière (1938), il choisit ainsi un vocabulaire néo-Renaissance traditionnel dans la région, maniant les frontons élevés à pas-de-moineau, les baies à meneaux ou en plein cintre (souvent superposées) et la polychromie brique et pierre, la monumentalité étant obtenue par l'usage de la symétrie des travées et d'une élévation à plusieurs niveaux. L'architecture de loisirs, elle, destinée aux rassemblements populaires, reçut un traitement particulier, recourant comme pour la piscine et le stade-parc aux recettes de l'Art déco : la salle des fêtes de Bruay (1930), de plain-pied, au toit en terrasse, fit ainsi appel à un rythme de dosserets cannelés évoquant la façade de la piscine; la salle des fêtes de Labuissière (décembre 1936) offrit une élévation plus originale, jouant sur l'alternance en hauteur et en couleur des travées et sur les horizontales des corniches et des appuis. Paul Hanote rechercha ainsi à exprimer de plusieurs façons une certaine dignité sociale à la collectivité, en exploitant le vocabulaire traditionnel et des schémas plus modernes, et en exécutant des bâtiments mêlant axialité, frontalité et monumentalité avec des moyens simples et économiques.

Ainsi, à travers l'exposé d'un contexte politique animé, on constate que la construction de bâtiments sportifs rend possible la promotion de la collectivité; d'une part, celle-ci montre son souci du bien-être des habitants, d'autre part elle fait valoir le dynamisme de sa gestion, en optant pour un parti architectural spectaculaire; enfin, les pratiques sportives administrent la preuve de la vitalité de la population et tout ce qui vient la valoriser augmente encore le prestige local.

La volonté qui a présidé à la réalisation de cet aménagement urbain mêlant sport et nature est emblématique de la tendance des années 1930 à l'affirmation de l'autorité municipale, au travers de grands projets à destination populaire. Le sport de masse et le loisir de tous sont ainsi mis à l'honneur, en préfiguration de l'œuvre du Front populaire. L'étude de cette période est ainsi tout à fait primordiale pour comprendre le processus d'individualisation des politiques sportives municipales. Si l'intérêt architectural de l'ensemble de Bruay-en-Artois est assez limité, la piscine, majestueusement ouverte sur $l^{\prime}$ horizon des terrils de la fosse $\mathrm{n}^{\circ} 6$, témoigne de conceptions hygiénistes et sociales originales.

\section{NOTES}

1. Bulletin officiel de la ville de Lyon, 31 août 1913, cité par Élisabeth Lê-Germain, « Lyon, une longueur d'avance », Le Sport et ses espaces, XIX ${ }^{e}$-XXe siècles, Paris, CTHS, t. 120-3, 1995.

2. Charles Toursel, Bruay-en-Artois et sa région de 1918 à 1945, édité par l'association des anciens élèves du lycée de Bruay-en-Artois, 1980, p. 60.

3. Henri Cadot (1864-1947) : il travailla très jeune à la mine et milita dans les mouvements socialistes et syndicalistes, fondant avec Émile Basly le syndicat des mineurs du Pas-de-Calais en 1889 (il le présida à partir de 1928). Il devint en 1893 directeur politique de La Tribune, organe du syndicat des mineurs où il s'employa à marquer l'importance des revendications ouvrières. Il intégra la SFIO en 1905 et, après trois échecs aux élections législatives, fut élu en 1914 dans le $4^{\mathrm{e}}$ 
arrondissement de Béthune. En 1919, il fut réélu député et devint maire de Bruay, charge qu'il occupa jusqu'en 1944. Entre 1930 et 1935, il exerça un mandat au Sénat mais ne fut pas renouvelé. Cf. Jean Maitron, Claude Pennetier, Dictionnaire biographique du mouvement ouvrier français, t. XXI, Paris, les Éditions ouvrières, 1984, p. 46. - Charles Toursel, op. cit., p. 33-35.

4. Le Pas-de-Calais, grand hebdomadaire régional de sensibilité républicaine (Archives départementales du Pas-de-Calais, G53/49), Journal de Bruay (id., G192/5).

5. Cette loi contraint les villes de plus de dix mille habitants à dresser un plan d'aménagement, d'embellissement et d'extension dans un délai maximal de trois ans.

6. Antoine Le Bas, Architectures du sport. Val de Marne-Hauts-de-Seine, Cahiers de l'Inventaire, 1991, introduction, par Françoise Hamon, p. 15.

7. Bruno Dumons, Gilles Pollet, Muriel Berjat, Naissance du sport moderne, Lyon, 1987, p. 66 et suiv. Vingt établissements de bain sont recensés en France en 1922, quand l'Allemagne en possède 1362.

8. En 1935, environ cent dix-huit villes françaises possédaient 169 installations nautiques homologuées par la fédération de natation sportive: petites dimensions $(25 \times 12 \mathrm{~m})$, forme rectangulaire, eau limpide et transparente. Thierry Terret, «L'eau, l'école et l'espace. Normes scolaires et pratiques de la natation au $\mathrm{XX}^{\mathrm{e}}$ siècle ", Le Sport et ses espaces, op. cit.

9. Jean-Paul Callède, « Notes d'architecture sportive. Le socialisme municipal des années trente à Bègles », Annales du Midi, t. 102, n 92, octobre-décembre 1990.

10. Édouard Herriot, Créer, Paris, Payot, 1920, 2 vol., cf. Jean-Paul Callède, art. cit., note 23.

11. Pierre Marie, Pour le sport ouvrier, éditions du Parti Socialiste SFIO, Paris, Librairie populaire, 1934. Il y était notamment question de concurrencer les organisations sportives bourgeoises et d'imiter les autres réalisations des communes socialistes.

12. Arch. dép. du Pas-de-Calais, bibliothèque.

13. Arch. mun. de Bruay, délibération du conseil municipal du 15 février 1935.

14. L'expression est tirée de l'article de Jean-Paul Callède, art. cit.

15. Le Pas-de-Calais, 10 mai 1936.

16. On sait aujourd'hui que la plupart des chantiers municipaux furent possibles grâce à des emprunts réalisés à des taux avantageux pour la commune auprès de la compagnie des mines (Ch. Toursel, op. cit., p. 184-195).

17. Le bassin d'initiation de Villeurbanne (1932) est de faible profondeur et de petite taille (18 x 5,5 m).

18. Ch. Toursel, op. cit., p. 325.

19. Le devis de Paul Hanote incluait un terrain de football, deux courts de tennis, une salle de culture physique et des tribunes. L'élément le plus intéressant de ce chantier achevé en 1933 est peut-être la salle de culture physique, avec une ossature en fer et une façade ornée de trois reliefs représentant des athlètes en action (réalisés par A. de Coninck, d'Anzin-Saint-Aubin).

20. DRAC Nord-Pas-de-Calais, Anne Lefebvre, dossier de protection au titre des monuments historiques, février 1997.

21. Bulletin municipal de Bruay-en-Artois, avril 1935, p. 12.

22. Ch. Toursel, op. cit., p. 319 et suiv.

23. Archives courantes des services techniques de la ville de Bruay-la-Buissière.

24. Bruno Dumons, Gilles Pollet, Muriel Berjat, Naissance du sport moderne, op. cit.

25. Réveil du Nord, 2 août 1936.

26. Archives courantes des services techniques de la ville de Bruay-la-Buissière. 
27. L'Architecte, L'Architecture d'aujourd'hui et La Construction moderne. L'Architecture d'aujourd'hui consacre dans ses numéros 9 (septembre 1935, p. 61-67) et 10 (octobre 1935) plusieurs articles dédiés aux piscines, la seconde livraison étant même dévolue presque exclusivement à ce genre d'équipement (les exemples de Bordeaux, Trouville, Villefranche-sur-Saône, Boran (plage) et Meaux-Trielport (plage), Wiesbaden et Paris (28 avenue d'Orléans, Croix-Catelan) sont abordés).

28. L'Architecte, janvier 1934, p. 8, planches 3-4.

29. Édifice à trois niveaux dominant le bassin extérieur, domination des horizontales, ailes basses en retour.

30. Paul François Hanote, né à Sin-le-Noble (Nord) le 30 juin 1873, décédé à Bruay-en-Artois le 9 février 1953, étudia à l'école des beaux-arts de Douai et obtint sa patente en 1897. Il fut lauréat d'un concours d'architecture et de charpente, reçut une médaille d'argent à l'exposition de Douai et fut expert près des tribunaux pour l'évaluation des dommages de guerre. Il était installé à Bruay-en-Artois en 1920 et participa aux chantiers de Bruay, Labuissière (salle des fêtes en 1936, hôtel de ville en 1938), Houdain (monument aux morts en 1921, école en 1923), Divion (monument aux morts en 1922). Il fut membre du conseil municipal de Bruay en 1941. Registres de Bruay-la-Buissière ; archives départementales du Pas-de-Calais, 10 R 1/4016 (cote provisoire) ; Ch. Toursel, op. cit., p. 184 et suiv.

31. René Hanote, son fils, naquit à Douai le 5 août 1897. Il fit ses études à Douai et travailla avec son père. Il fut reçu architecte en 1923 par le département du Pas-de-Calais, les communes de Bruay-en-Artois, Divion et Labuissière et l'office public des HBM de Bruay. Il devint en 1935 conseiller municipal de Rouen. Nath Imbert (dir.), Dictionnaire des contemporains, Paris, éd. Lajeunesse, 1938. - Le dossier aux Archives municipales de Rouen ne mentionne rien après 1939.

\section{RÉSUMÉS}

Pendant l'entre-deux-guerres, beaucoup de municipalités se mirent à rivaliser avec les organismes privés au sujet de l'urbanisme, considéré désormais comme un outil au service de l'amélioration du cadre de vie des habitants. Dans ce contexte, l'architecture sportive tient une place particulière, en particulier à l'époque où l'on tente de remettre le sport à l'honneur. L'exemple de la commune de Bruay-en-Artois (aujourd'hui Bruay-Labuissière dans le Pas-deCalais) et de la construction de sa piscine en plein air vers 1935-1936 illustre les débats électoralistes qui s'élevèrent à ce moment, en particulier sur le climat de lutte politique entre les socialistes (ici Henri Cadot, député et sénateur) et la puissante compagnie des mines de Bruay, pour s'assurer de la mainmise sur les habitants. En outre, la piscine est l'une des premières en France à s'intégrer au sein d'un ensemble sportif cohérent (parc, stade), dénotant ainsi le souci des concepteurs de lier sport et santé publique. Enfin, sur ce chantier, l'architecte Paul Hanote (1879-1953) se montre comme un tenant de l'Art déco (goût pour les horizontales, la symétrie, les lignes droites) et applique les principes fonctionnalistes énoncés dans les revues spécialisées autour de l'utilisation de matériaux modernes et simples mais privilégiant le monumentalisme.

This article means to describe how, in the town of Bruay-en-Artois (Pas-de-Calais), during the interwar years, the local authority began to consider urban planning as a way to enlighten people's life, and how sport architecture and sport practice held a place in this evolution. On the one hand, through the description of this construction period (1935-1936), at a time when sport turned to be a matter for debate and a way to win people over, one can have an idea of the 
rivalries between local_socialists, such as mayor and deputy Henri Cadot, and powerful coal companies, for running local policies. On the other hand, this building, nestled in a stadium and a huge garden, is one of the first in France to take into account both public health and physical education, and symbolizes the growing concern about public health. Eventually, since this swimming pool displays many Art Déco shapes (taste for horizontals, symmetry, straight lines), one can relevantly underline that the architect, Paul Hanote (1879-1953), got involved in an intellectual movement discussing on the functionalist architecture and the use of modern and simple materials, which can nonetheless conveyed monumentality and dignity.

In der Zwischenkriegszeit entwickelt sich eine urbane Politik, die auf die Verbesserung des Lebensrahmens der Stadtbewohner ausgerichtet ist. Besonders in dieser Zeit der allgemeinen Begeisterung für Sport erweist sich der Bau von Sportanlagen als eine ganz bedeutsame Aufgabe der Stadtverwaltungen im Wettbewerb mit privaten Trägern. Das Beispiel des Freibads von Bruay in den Jahren 1935-1936 führt alle politischen Interessen vor Augen. Aufgrund des damaligen Wahlkampfes gibt der Bau Anlass zu dauernden Auseinandersetzungen zwischen den sozialistischen Behörden und der mächtigen Compagnie des Mines de Bruay ( Bergwerkgesellschaft), die beiderseits die Zustimmung der Einwohner erringen wollen. Das Schwimmbad von Bruay, das zu den ersten Experimenten dieser Art in Frankreich zählt, wird als eine Erholungsanlage mit Park und Stadium gebaut, wo sowohl Sport als auch öffentliche Gesundheit gefördert werden sollen. Der Architekt Paul Hanote (1879-1953) entschließt sich für Symmetrie, waagerechte und gerade Linien je nach dem Art Déco Stil. Er übernimmt andererseits die in den Fachzeitschriften geäußerten Prinzipien des Funktionalismus : zwar moderne einfache Materialien verwenden, aber monumentale Formen bevorzugen.

\section{INDEX}

Index chronologique : XXe siècle, époque contemporaine

Mots-clés : piscine, Art déco, socialisme

Keywords : swimming pool, Art Deco, socialism

Schlüsselwörter : Schwimmbad, Art déco, Sozialismus

\section{AUTEUR}

\section{MARC VERDURE}

Marc Verdure, archiviste paléographe, est l'auteur d'une thèse d'École des chartes, Recherches sur le monastère byzantin Saint-Jean-Prodrome à Serrès, XII ${ }^{e}-\mathrm{XV}^{e}$ siècle (inédite). Également conservateur du patrimoine, il travaille aujourd'hui au conseil général du Pas-de-Calais (mission patrimoine, musées, mémoire). 